\title{
Two-stage Beamformer Design via Deterministic Equivalents
}

\author{
Hossein Asgharimoghaddam and Antti Tölli \\ Centre for wireless communications (CWC), University of Oulu, Oulu, Finland \\ \{hossein.asgharimoghaddam, antti.tolli\}@oulu.fi
}

\begin{abstract}
Complexity reduction of optimal linear receiver is considered in a scenario where both the number of single antenna user equipments (UEs) $K$ and base station (BS) antennas $N$ are large. Two-stage beamforming (TSB) greatly alleviates the high implementation complexity of large scale multiantenna receiver by concatenating a statistical outer beamformer (OBF) with an instantaneous inner beamformer (IBF) design. Using asymptotic large system analysis, we propose a novel TSB method that adjusts the dimensions of user specific OBF matrices based on the projection of the optimal minimum mean square error (MMSE) vectors into the beam domain. The beam domain is first divided into $S$ narrow sectors such that each sector contains $D$ DFT beams. Then, so called deterministic equivalents are computed for the amplitude-projection of the optimal MMSE vectors into each sector in asymptotic regime where $N, K$ and $D$ grow large with a non-trivial ratio $N / K=C$ and $N / D=S$. Given the approximations for the sector specific values, the structure and dimension of each UE specific OBF vector are optimized based on the statistical channel properties and the amount of overlap among users in angular domain. The numerical analysis shows that the attained SINR values closely follow the optimal MMSE receiver while the computational burden is greatly reduced.
\end{abstract}

\section{INTRODUCTION}

High spatial utilization is a promising approach to meet the significant spectral efficiency enhancements required for $5 \mathrm{G}$ cellular networks. In general, this is achieved by using a large number of antennas $N$ at the base stations (BSs) to serve a large number of user equipments (UEs) $K$ on the same frequency-time resources. However, such large dimensions of the channel matrices pose challenges on computational complexity and hardware costs. A promising solution to these problems lies in the concept of two-stage beamforming (TSB), which concatenates an outer-beamformer (analog/digital) with an inner beamformer/receiver.

Joint spatial division and multiplexing (JSDM) is introduced in [1] for a downlink scenario wherein a statistical OBF matrix creates multiple virtual sectors. Exploiting the similarity among covariance matrices of co-located UEs, the authors in [1] propose to group UEs based on their statistical properties. Then, the OBF matrix is designed based on the eigenvectors of group-specific covariance matrices. The performance of such a system depend on group-formation, and cross-sector interference management [2], [3]. The authors in [4], [5] study JSDM-based TSB in a downlink system to maximize the weighted sum-rate. It is observed that the

This work has been supported in part by the Academy of Finland 6Genesis Flagship (grant no. 318927). reduced spatial dimensions results in significant inter-sector interference leakage as the number of UEs $K$ increases. This issue is addressed in [5] by coordination of interference among sectors. Similar performance degradation appears in the equivalent uplink problem where the work in [6] mitigates the effects of inter-group interference using layered belief propagation detector.

In this paper, we consider uplink of a single-cell system wherein $K$ single-antenna UEs communicate with a BS equipped with $N$ antennas. In this case, it is well-known that linear minimum mean square error (MMSE) receiver attains the maximum signal-to-interference-plus-noise ratios (SINRs) [7]. Motivated by this observation, a novel JSDMATSB method is proposed that adjusts the dimensions of UEspecific OBF matrices based on the projection of the MMSE vectors into the beam domain. To this end, the angular-domain is divided into $S$ fixed narrow-sectors such that each sector contains $D<<N$ DFT beams. Then, so-called deterministic equivalents [8] are computed for the amplitude projection of MMSE vectors into each sector via asymptotic analysis in a regime, where $N, K$ and $D$ grow large with a non-trivial ratio $N / K=c$ and $N / D=S$. The deterministic equivalents provide tight approximations for the considered metrics in finite-dimensional problems while those depend only on the statistical CSI [8]. The OBF matrix of each UE is obtained by concatenating the sectors whose AP-MMSES values are larger. As a result, the OBF matrices adopt the MMSE strategy, and adjust the direction and the number of sectors for each UE based on the level of multiple access interference while relying solely on statistical CSI. The inner-receiver for a UE $\mathrm{k}$ is designed, as in the conventional TSB methods, based on the resulting reduced dimensional channel matrix of size $D_{k} \times K$. The numerical analysis shows that the attained per-UE rates closely follow the rate of optimal MMSE receiver. Also, it is observed that the dimension $D_{k}$ depends on the angular position of UE $k$, system load, UEs' angular spread, UEs' powers, and the desired bound on performance degradation.

\section{Problem Statement}

\section{A. System Model}

We consider uplink of a single-cell multi-user large-scale MIMO system, where a single base station (BS) with $N$ antenna elements serves $K<N$ single-antenna user terminals (UE). Under this convention and assuming narrow-band transmission, we define $\mathbf{h}_{k} \in \mathbb{C}^{N}$ as the channel between the 


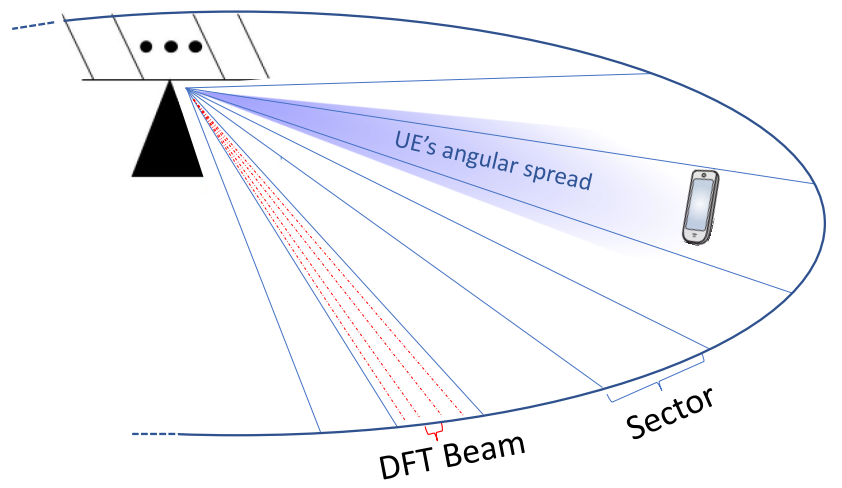

Fig. 1. Illustration of sectorized angular domain.

$\mathrm{BS}$ and UE $k$. Then, the received signal of UE $k$ at BS can be expressed as

$$
y_{k}=\mathbf{w}_{k}^{\mathrm{H}} \mathbf{h}_{k} x_{k}+\sum_{i \backslash k} \mathbf{w}_{k}^{\mathrm{H}} \mathbf{h}_{i} s_{i}+\mathbf{w}_{k}^{\mathrm{H}} \mathbf{n}
$$

where the first term is the desired signal and the second term represents intra-cell interference. The vector $\mathbf{w}_{k} \in \mathbb{C}^{N}$ denotes the receiver vector of UE $k$. The zero mean, unit variance data symbol intended to UE $k$ is denoted by $x_{k}$, and is assumed to be independent across UEs. Zero-mean white Gaussian noise at the receiver is denote by $\mathbf{n} \sim$ $\mathcal{C N}\left(0, \sigma^{2} \mathbf{I}_{N}\right)$. The MMSE receiver for a UE $k$ is given as $\mathbf{w}_{k}^{\star}=\left(\sum_{j \backslash k} p_{j} \mathbf{h}_{j} \mathbf{h}_{j}^{\mathrm{H}}+\sigma^{2} \mathbf{I}_{N}\right)^{-1} \mathbf{h}_{k}$ with $p_{j}$ being the transmit power of UE $j$. The superscript ( $)^{\star}$ indicates the optimality of the MMSE receiver. Nevertheless, the implementation of large scale MMSE receiver is not feasible with large antenna arrays due to computational complexity constraints. Given a limited angular spread 1 it is possible to do receive-processing in a smaller dimensional space than $N$. To this end, we first need to introduce a statistical model for the channel vectors.

\section{B. Channel Model}

The channel from BS to UE $k$ is modelled as $\mathbf{h}_{k}=\Theta_{k}^{1 / 2} \mathbf{z}_{k}$ where $\mathbf{z}_{k} \in \mathbb{C}^{N}$ represents small-scale fading and has i.i.d, zero-mean, unit-variance complex entries. The matrix $\boldsymbol{\Theta}_{k} \in$ $\mathbb{C}^{N \times N}$ accounts for the UE specific channel correlation at the BS. The pathloss due to large scale fading is implicitly considered in the correlation matrix unless otherwise stated. In the latter case, pathloss values are explicitly declared by expressing the correlation matrix as $a_{k}^{2} \boldsymbol{\Theta}_{k}$ where $a_{k}^{2}$ accounts for pathloss from the BS to UE $k$.

\section{Beamformer design}

The beamforming vector of a UE $k$ is presented as $\mathbf{w}_{k}=$ $\mathbf{B}_{k} \mathbf{v}_{k}$, where we'd like to design outer-beamfomer (OBF) $\mathbf{B}_{k} \in \mathbb{C}^{N \times D_{k}}$ based on statistical CSI in order to decrease the complexity of inner-receiver $\mathbf{v}_{k} \in \mathbb{C}^{D_{k}}$. Here, $\left\{D_{k}\right\}_{\forall k}$ are UE dependent design parameters, which trade-off performance and

\footnotetext{
${ }^{1}$ In a typical cellular configuration with a tower-mounted BS and no significant local scattering, the propagation between the BS antennas and any given UE is expected to be characterized by the local scattering around the UE. This results in UE's signal to arrive at BS from a limited angular spread.
}

complexity of obtaining the inner-receivers. To do so, we divide the beam-domain into $S$ fixed narrow sectors $\left\{\mathbf{S}_{i}\right\}_{1<i<S}$ as shown in Fig. 11 Let $\mathbf{U}$ be the $N \times N$ unitary matrix the columns of which are DFT vectors/beams $\left\{\mathbf{u}_{j}\right\}_{1 \leq j \leq N}$. Each sector contains $D$ DFT beams, i.e., $\mathbf{S}_{i}=\left\{\mathbf{u}_{j}, j \in\right.$ $\{(i-1) \times D+1, \ldots,(i-1) \times D+D\}\}$. Since MMSE vector is the optimal receiver for the considered system model, we propose to form the OBFs based on projection of the optimal MMSE vectors into each sector. The projection of MMSE vector of UE $k$ into a sector $\mathbf{S}_{i}$ is given as $\mathbf{S}_{i}^{\mathrm{H}} \mathbf{w}_{k}^{\star}$, and thus, the normalized squared norm of this projection, denoted by $\omega_{k, i}$, is given as

$$
\omega_{k, i}=\frac{1}{N} \mathbf{h}_{k}^{\mathrm{H}} \boldsymbol{\Sigma}_{k} \mathbf{S}_{i} \mathbf{S}_{i}^{\mathrm{H}} \boldsymbol{\Sigma}_{k} \mathbf{h}_{k}
$$

where $\boldsymbol{\Sigma}_{k}=\left(\sum_{j \backslash k} p_{j} \mathbf{h}_{j} \mathbf{h}_{j}^{\mathrm{H}}+\mathbf{I}_{N}\right)^{-1}$. Given approximations for $\omega_{k, i}$ values, the OBF for UE $k$ can be attained by selecting the sectors that have larger projection norm. This ensures that the inner-receiver has enough information to yield SINR values close to the optimal MMSE ones. Under this convention, the SINR of a UE is attained via inner-receiver by processing signals received within a vector space of size $D_{k}<N$. In deriving the approximations for $\omega_{k, i}$ values, we use results from random matrix theory that allows approximating functional of the random matrices by deterministic quantities [8]. These quantities depend only on the underlying statistical properties, and yield precise approximations for practical problems of finite dimensions. The result of this analysis is presented in the following section.

\section{LARGE SYSTEM ANALYSIS}

In deriving the large system analysis, the following assumptions (widely used in the literature) are made to properly define the growth rate of system dimensions.

Assumption 1. As $N \rightarrow \infty, 0<\frac{N}{K}<\infty$, and $0<\frac{N}{S}<\infty$.

Assumption 2. The spectral norm of $\boldsymbol{\Theta}_{k}$ is uniformly bounded as $N \rightarrow \infty$, i.e., $\lim \sup _{N \rightarrow \infty} \max _{\forall k}\left\{\left\|\boldsymbol{\Theta}_{k}\right\|\right\}<\infty$.

In order to ensure that the total power in the system does not grow unbounded as the number of UEs grow large, we normalize UEs' powers by the number of antennas $N$. Also, without loss of generality, the Gaussian noise variance is assumed to be one. Following the same approach as in [9], deterministic equivalents for $\omega_{k, i}$ terms can be derived in terms of statistical CSI. The results are summarized in the following theorem.

Theorem 1. Under Assumptions 1,2 the following holds almost surely

$$
\omega_{k, i}-\bar{\omega}_{k, i} \rightarrow 0
$$

where the values of $\bar{\omega}_{k, i}$ can be evaluated as

$$
\left[\bar{\omega}_{1, i}, \ldots, \bar{\omega}_{K, i}\right]=\left(\mathbf{I}_{K}-\mathbf{L}\right)^{-1} \mathbf{b}_{i}, \forall i \in\{1, \ldots, S\}
$$

where

$$
[\mathbf{L}]_{i, j}=\frac{1}{N} \frac{\operatorname{tr}\left(\boldsymbol{\Theta}_{i} \mathbf{T} \Theta_{j} \mathbf{T}\right)}{\left(1 / \bar{p}_{j}+\bar{m}_{j}\right)^{2}},
$$


and

$$
\mathbf{b}_{i}=\left[\frac{1}{N} \operatorname{tr}\left(\boldsymbol{\Theta}_{1} \mathbf{T} \mathbf{S}_{i} \mathbf{S}_{i}^{\mathrm{H}} \mathbf{T}\right), \ldots, \frac{1}{N} \operatorname{Tr}\left(\mathbf{\Theta}_{K} \mathbf{T} \mathbf{S}_{i} \mathbf{S}_{i}^{\mathrm{H}} \mathbf{T}\right)\right]
$$

with $\mathbf{T}$ given by

$$
\mathbf{T}=\left(\frac{1}{N} \sum_{j \in \mathcal{U}} \frac{\bar{p}_{j} \boldsymbol{\Theta}_{j}}{1+\bar{p}_{j} \bar{m}_{j}}+\mathbf{I}_{N}\right)^{-1},
$$

and $\bar{m}_{j}, \forall j \in \mathcal{U}$ are given as the fixed-point solution of $\bar{m}_{j}=$ $\frac{1}{N} \operatorname{tr}\left(\boldsymbol{\Theta}_{j} \mathbf{T}\right), \forall j \in \mathcal{U}$.

Proof: The proof is given in Appendix A

The results of the theorem yield approximations for $\omega_{k, i}, k \in \mathcal{U}, i \in\{1, \ldots, S\}$ values in finite regime. The results are utilized in the following to propose algorithms for obtaining OBF matrices.

\section{Algorithms fOR Designing TWO-STAGE BEAMFORMERS}

Given approximations for $\omega_{k, i}$ values, the OBF for UE $k$ can be attained by selecting the sectors that have larger projection norm, i.e., $\mathbf{B}_{k}=\left\{\mathbf{S}_{i}\right\}_{i \in \mathcal{B}_{k}}$ where $\mathcal{B}_{k}$ holds the indices of selected sectors. The received signal for UE $k$ after applying $\mathrm{OBF}$ is given as

$$
\mathbf{B}_{k}^{\mathrm{H}} \mathbf{y}=\mathbf{B}_{k}^{\mathrm{H}} \mathbf{H} \mathbf{x}+\mathbf{B}_{k}^{\mathrm{H}} \mathbf{n}
$$

where $\mathbf{H}=\left[\mathbf{h}_{1}, \ldots, \mathbf{h}_{K}\right]$, and $\mathbf{x}=\left[x_{1}, \ldots, x_{K}\right]^{\mathrm{T}}$. The innerreceiver of UE $k$ applies a MMSE vector based on the $D_{k} \times K$ equivalent channel given by $\mathbf{B}_{k}^{\mathrm{H}} \mathbf{H}$. Since we have $D_{k}=\left|\mathcal{B}_{k}\right| \times D$, the complexity of inner-receiver is determined by the cardinality of set $\mathcal{B}_{k}$. For a given UE $k$, we propose to select the sectors whose $\bar{\omega}_{k, i}$ values are larger than $\delta \max \left(\bar{\omega}_{k, 1}, \ldots, \bar{\omega}_{k, S}\right)$. The parameter $0 \leq \delta \leq 1$ trades off the complexity and performance. Larger $\delta$ values yield smaller $D_{k}$ values but also degrades the performance. These steps are summarized in Algorithm 1 .

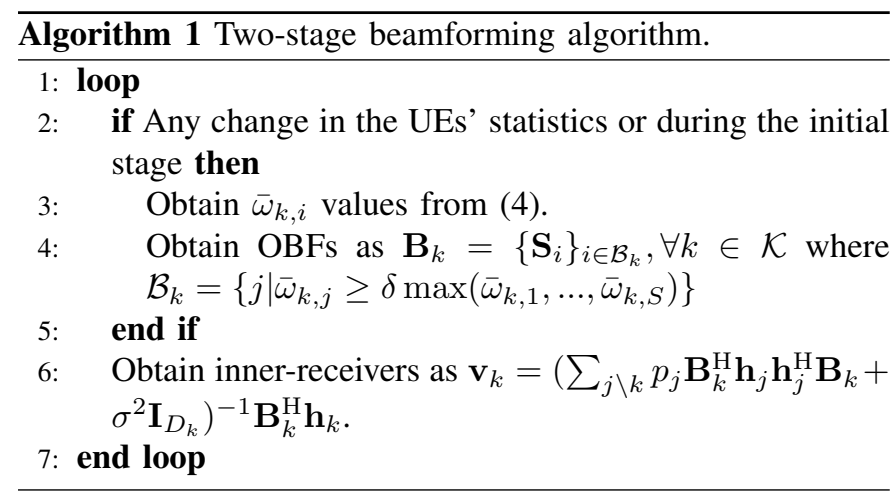

Concerning the complexity analysis, we notice that the evaluation of inner-receiver $\mathbf{v}_{k}$ involves a matrix inversion of size $D_{k} \times D_{k}$ with a complexity in the order of $\mathcal{O}\left(D_{k}^{3}\right)$. Due to the limited angular spread of UEs' signals, $D_{k}$ values are expected to be much smaller than $N$. Concerning the calculation of approximate $\bar{\omega}_{k, i}$ values, we notice that $\bar{\omega}_{k, i}$ values in Step 3 of the algorithm are updated only when there are sufficient changes in CSI statistics, which vary at a much slower rate
90

$$
120
$$$$
6 \times 10^{-3}
$$

60

150

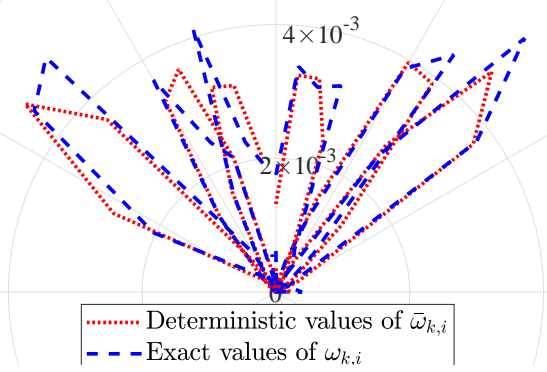

30

Fig. 2. The values of $\bar{\omega}_{k, i}$ and $\omega_{k, i}$ for 5 selected UEs, $N=225, K=135$, $S=45$.

than the fading CSI. The computation of approximate $\bar{\omega}_{k, i}$ values requires matrix inversion in $\left(\mathbf{I}_{K}-\mathbf{L}\right)^{-1}$, and evaluation of $\left\{\bar{m}_{k}\right\}$ values. The complexity of evaluating the former one is of order $\mathcal{O}\left(K^{3}\right)$. The latter one is evaluated via a fixed point iteration with complexity of $\mathcal{O}\left(N^{3}\right)$ per-iteration.

\section{Numerical Analysis}

Monte Carlo simulations are now used to validate the performance of the proposed solution. By assuming a diffuse 2-D field of isotropic scatterers around the receiver [10], the correlation matrix for an antenna element spacing of $\Delta$ is given by

$$
\left[\boldsymbol{\Theta}_{k}\right]_{j, i}=\frac{a_{k}^{2}}{\varphi_{k}^{\max }-\varphi_{k}^{\min }} \int_{\varphi_{k}^{\min }}^{\varphi_{k}^{\max }} e^{i \frac{2 \pi}{w} \Delta(j-i) \cos (\varphi)} \mathrm{d} \varphi
$$

where waves arrive with an angular spread $\Delta \varphi$ from $\varphi_{\min }$ to $\varphi_{\max }$. The wavelength is denoted by $w$, and the antenna element spacing is fixed to half the wavelength $\Delta=1 / 2 w$. The UEs are distributed over a circle of radius $300 \mathrm{~m}$ between angular position $\frac{\pi}{6}$ to $\frac{5 \pi}{6}$. The angular separation between UEs are the same and equal to $\frac{2 \pi}{3} \frac{1}{K}$. The angular spread $\Delta \varphi$ is the same for all UEs and equal to $\pi / 10$. Thus, increasing the number of UEs results in an increase in overlap among UEs' signals angle-of-arrivals (AOAs). The number of antennas at BS is fixed to $N=225$, and the angular domain is divided into $S=45$ sectors. The effect of pathloss and additive noise is captured in received signal to noise ratio (SNR) at an antenna element of BS. The SNR is denoted by $\rho$ in the following.

In order to validate the large system analysis, Fig. 2 shows the exact values of $\omega_{k, i}$ and the deterministic equivalents $\bar{\omega}_{k, i}$ for 5 selected UEs, and a given random realization of smallscale fading. The number of UEs $K$ is equal to 135 . It can be seen that the values of deterministic $\bar{\omega}_{k, i}$ closely follows the exact ones $\omega_{k, i}$. The OBF matrices in Algorithm 1 are designed based on these accurate approximations. Thus, the spatial filtering is expected to reduce the dimensions of innerreceivers with minimal performance degradation.

Fig. 3 illustrates the trade off between complexity and performance in Algorithm 11. The upper and lower plots in the figure show the averaged number of beams allocated per 
$\mathrm{UE}$ and attainable rates in $\mathrm{b} / \mathrm{s} / \mathrm{Hz} / \mathrm{UE}$, respectively, versus $\delta$ values. The results are presented for the cases with the number of UEs equal to 135 and 225. The attainable rate using Algorithm 1 is titled as OBF-MMSE in the figure. Also, the rates of optimal MMSE receiver and matched filtering are presented as benchmarks. As can be seen from the figure, the number of beams per UE decreases as $\delta$ value increases. The parameter $\delta$ adjusts the number of beams that are passed to the inner-receiver. A higher value of $\delta$ neglects more beams with small AP-MMSE values. Setting $\delta=0.1$ as an example, cuts off the sectors whose AP-MMSE values are less than one-tenth of the maximum value. It can be seen that at point $\delta=0.1$, the gap to the optimum rate is small. Also, the number of beams per UE is near $N / 4$. Thus, at $\delta=0.1$ a proper trade off between the performance and complexity is achieved.

In Fig. 4, the attainable rate in $\mathrm{b} / \mathrm{s} / \mathrm{Hz} / \mathrm{UE}$ along with corresponding averaged number of beams per UE in Algorithm 1 is plotted versus load $K / N$. The results are presented for the cases with $\delta$ equals to 0.01 and 0.1 . Interestingly the gap to the optimal rate is almost fixed for a given value of $\delta$ over the whole range of load $K / N$. The larger values of $\delta$ yield a larger gap. It can be seen that the number of beams per UE increases as load of the system increases. This is due to the fact that larger load results in stronger multiple access interference. Thus, in order to keep the performance degradation within a given limit, a larger number of degrees of freedom is needed in the inner-receivers to mitigate the interference. In an alternative presentation, the number of beams allocated to each UE is plotted versus UEs' angular positions in Fig. 5. The value of parameter $\delta$ is fixed to 0.1, and the results are plotted for various number of UEs. As mentioned earlier the higher load generally needs a larger number of beams to keep a certain performance degradation. The other observation is that the number of allocated beams is larger for UEs residing in front of antenna array, while UEs in sides of the array needs smaller number of beams. This is due to the fact that the signal of UEs residing in sides of the
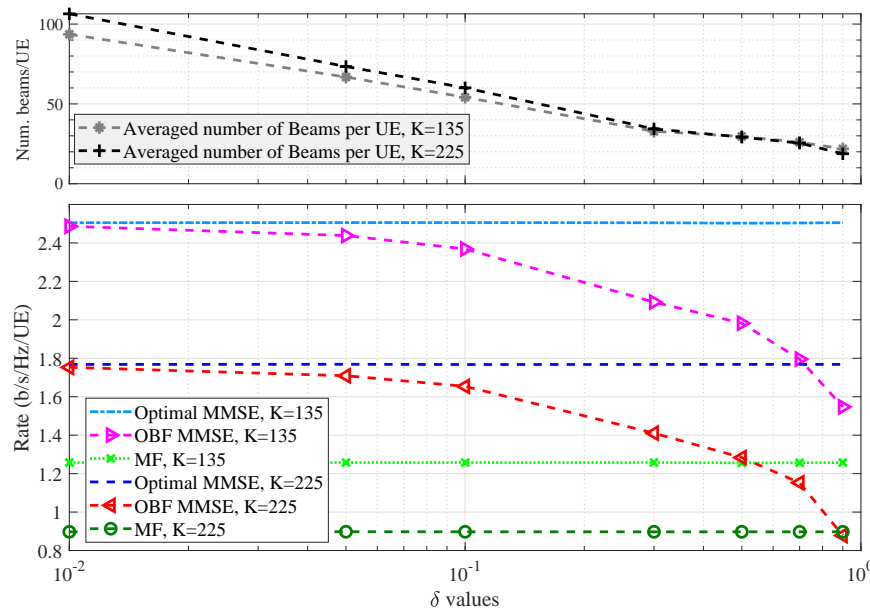

Fig. 3. Rate and the number of beams per UE vs. $\delta, N=225, S=45$, $\rho=10$.

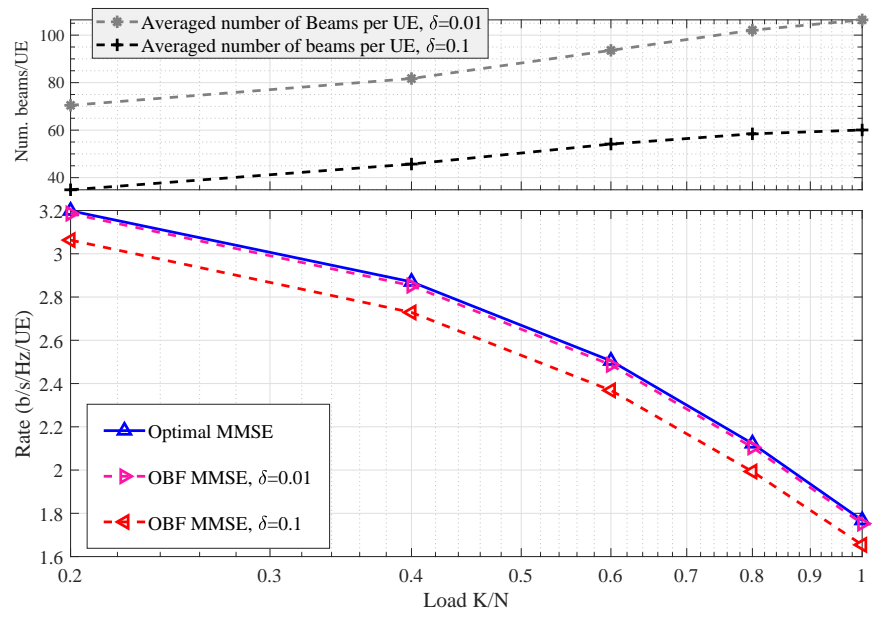

Fig. 4. Rate and the number of beams per UE vs. load $K / N, N=225$, $S=45, \rho=10$.

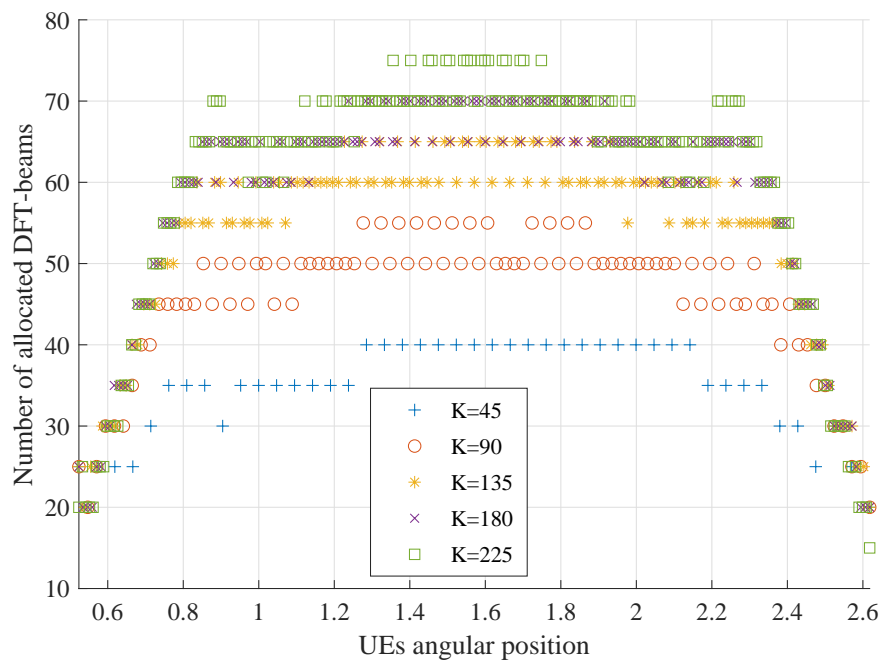

Fig. 5. The number of allocated beams vs. angular position of UEs, $\delta=0.1$, $N=225, S=45, \rho=10$.

array are less interfered. Also, the DFT beams become more dense in front of the array while the resolution of DFT beams decreases towards the sides of the array.

\section{CONCLUSions}

Based on large system analysis, a novel TSB method was proposed that adjusts the dimensions of UE-specific OBF matrices based on the projection of the optimal MMSE vectors into the beam domain. This approach takes the multi-access interference into account when designing OBF, and thus, yields an optimal selection of sectors for a given UE. This allowed us to study the optimal window-sizes $\left|\mathcal{B}_{k}\right|$ given a certain performance degradation. It was observed that the window-size in average increases as the load of the system grows large, i.e., as multiple-access interference increases. Also, the numerical analysis showed that the UEs residing in the sides of the antenna array need smaller window-sizes, which is due to lower interference and lower resolution of 
DFT beams in the sides of the array. It was shown that the attained SINR values based on the proposed approach closely follow the optimal MMSE receiver while the computational burden of obtaining inner-beamformer is greatly reduced.

\section{APPENDIX A \\ PROOF OF THEOREM 1}

In the derivation of large system analysis, we use wellknown lemmas including trace lemma [11, Lemma 2.6], [8. Theorem 3.4] along with rank-1 perturbation lemma [12. Lemma 2.6], [8, Theorem 3.9]. The former one shows asymptotic convergence of $\mathbf{x}^{\mathrm{H}} \mathbf{A} \mathbf{x}-\frac{1}{N} \operatorname{Tr} \mathbf{A} \rightarrow 0$ when $\mathbf{x} \in \mathbb{C}^{N}$ has i.i.d entries with zero mean, variance of $\frac{1}{N}$ and independent of $\mathbf{A}$. The latter one states that addition of rank-1 matrix $\mathbf{x x}^{\mathrm{H}}$ to the random Gram matrix $\mathbf{X X} \mathbf{X}^{\mathrm{H}}$ does not affect trace $\frac{1}{N} \operatorname{Tr}\left(\mathbf{X X}^{\mathrm{H}}+\mathbf{I}_{N}\right)$ term in the large dimensional limit. The formal presentation of these lemmas are given in [11], [12].

Starting from amplitude projection in (2), we apply trace lemma, along with rank-1 perturbation lemma to get

$$
\omega_{k, i}-\frac{1}{N} \operatorname{tr}\left(\boldsymbol{\Theta}_{k} \boldsymbol{\Sigma} \mathbf{S}_{i} \mathbf{S}_{i}^{\mathrm{H}} \boldsymbol{\Sigma}\right) \stackrel{N \rightarrow \infty}{\longrightarrow} 0
$$

almost surely, where $\boldsymbol{\Sigma}=\left(\sum_{j} p_{j} \mathbf{h}_{j} \mathbf{h}_{j}^{\mathrm{H}}+\mathbf{I}_{N}\right)^{-1}$. From matrix identities [13], we know that $\partial \mathbf{Y}^{-1} / \partial x=$ $-\mathbf{Y}^{-1}(\partial \mathbf{Y} / \partial x) \mathbf{Y}^{-1}$ with $\mathbf{Y}$ being a matrix depending on variable $x$. Thus, the above trace term can be written equivalently as

$$
\frac{1}{N} \operatorname{tr}\left(\boldsymbol{\Theta}_{k} \boldsymbol{\Sigma} \mathbf{S}_{i} \mathbf{S}_{i}^{\mathrm{H}} \boldsymbol{\Sigma}\right)=\left.\frac{\partial}{\partial x} m_{k, i}(z, x)\right|_{x=0, z=-1}
$$

where

$$
m_{k, i}(z, x)=\frac{1}{N} \operatorname{tr}\left(\boldsymbol{\Theta}_{k}\left(\sum_{j} p_{j} \mathbf{h}_{j} \mathbf{h}_{j}^{\mathrm{H}}-z \mathbf{I}_{N}-x \mathbf{S}_{i} \mathbf{S}_{i}^{\mathrm{H}}\right)^{-1}\right) .
$$

The term $m_{k, i}(z, x)$ is the Stieltjes transforms of a measure. It is shown in [9, Theorem 1], where under Assumption 1, 2. and for $z \in \mathbb{C} \backslash \mathbb{R}^{+}, x \in \mathbb{R}^{-}$, these Stieltjes transforms have deterministic equivalents such that

$$
m_{k, i}(z, x)-\bar{m}_{k, i}(z, x) \stackrel{N \rightarrow \infty}{\longrightarrow} 0
$$

almost surely, where the deterministic equivalents $\bar{m}_{k, i}(z, x)$ are given as the solutions of the following fixed-point iterations

$$
\bar{m}_{k, i}(z, x)=\frac{1}{N} \operatorname{tr}\left(\boldsymbol{\Theta}_{k} \mathbf{T}_{i}(z, x)\right), \forall k \in \mathcal{U}, i \in\{1, \ldots, S\}
$$

where

$$
\mathbf{T}_{i}(z, x)=\left(\frac{1}{N} \sum_{j=1}^{K} \frac{p_{j} \boldsymbol{\Theta}_{j}}{1+p_{j} \bar{m}_{j, i}(z, x)}-x \mathbf{S}_{i} \mathbf{S}_{i}^{\mathrm{H}}-z \mathbf{I}_{N}\right)^{-1}
$$

As the result, from 10, 111, and (13), we get

$$
\omega_{k, i}-\bar{m}_{k, i}^{\prime} \stackrel{N \rightarrow \infty}{\longrightarrow} 0
$$

where $\bar{m}_{k, i}^{\prime}=\left.\bar{m}_{k, i}^{\prime}(z, x)\right|_{x=0, z=-1}$ with $\bar{m}_{k, i}^{\prime}(z, x) \triangleq$ $\frac{\partial}{\partial x} \bar{m}_{k, i}(z, x)$. The values of $\bar{m}_{k, i}^{\prime}$ can be evaluated by taking derivative of $\bar{m}_{k, i}(z, x)$ in (14), and evaluating the derivative at point $(x=0, z=-1)$. In doing so, we get

$$
\bar{m}_{k, i}^{\prime}=\frac{1}{N} \operatorname{tr}\left(\boldsymbol{\Theta}_{k} \mathbf{T}_{i}^{\prime}\right), \forall k \in \mathcal{U}, i \in\{1, \ldots, S\}
$$

where $\mathbf{T}_{i}^{\prime}=\left.\mathbf{T}_{i}^{\prime}(z, x)\right|_{x=0, z=-1}$, or equivalently

$$
\mathbf{T}_{i}^{\prime}=\mathbf{T}\left(\frac{1}{N} \sum_{j \in \mathcal{U}} \frac{p_{j}^{2} \Theta_{j} \bar{m}_{j, i}^{\prime}}{\left(1+p_{j} \bar{m}_{j}\right)^{2}}+\mathbf{S}_{i} \mathbf{S}_{i}^{\mathrm{H}}\right) \mathbf{T}
$$

where $\mathbf{T}=\mathbf{T}_{i}(-1,0)$, and $\bar{m}_{j}=\bar{m}_{j, i}(-1,0)$. Since $\bar{m}_{k, i}^{\prime}=\frac{1}{N} \operatorname{Tr}\left(\Theta_{k} \mathbf{T}_{i}^{\prime}\right)$ with $\mathbf{T}_{i}^{\prime}$ given by $(18)$, we get a system of equation to evaluate $\bar{m}_{k, i}^{\prime}$ as $\left[\bar{m}_{1, i}^{\prime}, \ldots, \bar{m}_{K, i}^{\prime}\right]=\left(\mathbf{I}_{K}-\mathbf{L}\right)^{-1} \mathbf{b}_{i}$ with $\mathbf{b}_{i}$ and $\mathbf{L}$ defined as in (6) and (7), respectively, which completes the proof of the theorem.

\section{REFERENCES}

[1] A. Adhikary, J. Nam, J. Y. Ahn, and G. Caire, "Joint spatial division and multiplexing: The large-scale array regime," IEEE Transactions on Information Theory, vol. 59, no. 10, pp. 6441-6463, Oct 2013.

[2] Y. Xu, G. Yue, N. Prasad, S. Rangarajan, and S. Mao, "User grouping and scheduling for large scale mimo systems with two-stage precoding," in 2014 IEEE International Conference on Communications (ICC), June 2014, pp. 5197-5202.

[3] J. Nam, A. Adhikary, J. Ahn, and G. Caire, "Joint spatial division and multiplexing: Opportunistic beamforming, user grouping and simplified downlink scheduling," IEEE Journal of Selected Topics in Signal Processing, vol. 8, no. 5, pp. 876-890, Oct 2014.

[4] A. Arvola, A. Tlli, and D. Gesbert, "Two-layer precoding for dimensionality reduction in massive mimo," in 2016 24th European Signal Processing Conference (EUSIPCO), Aug 2016, pp. 2000-2004.

[5] A. Padmanabhan and A. Tlli, "Interference management via user clustering in two-stage precoder design," in 2018 IEEE 19th International Workshop on Signal Processing Advances in Wireless Communications (SPAWC), June 2018, pp. 1-5.

[6] T. Takahashi, A. Tolli, S. Ibi, and S. Sampei, "Layered belief propagation for low-complexity large mimo detection based on statistical beams," in ICC 2019 - 2019 IEEE International Conference on Communications (ICC), May 2019, pp. 1-6.

[7] D. Tse and P. Viswanath, Fundamentals of Wireless Communication. Cambridge University Press, 2005.

[8] R. Couillet and M. Debbah, Random Matrix Methods for Wireless Communications. Cambridge University Press, 2011.

[9] S. Wagner, R. Couillet, M. Debbah, and D. Slock, "Large system analysis of linear precoding in correlated MISO broadcast channels under limited feedback," IEEE Transactions on Information Theory, vol. 58, no. 7, pp. 4509-4537, July 2012.

[10] W. C. Jakes, Microwave Mobile Communications, 1st ed. Wiley-IEEE Press, 1994.

[11] Z.-D. Bai and J. W. Silverstein, "No eigenvalues outside the support of the limiting spectral distribution of large-dimensional sample covariance matrices," Annals of probability, pp. 316-345, 1998.

[12] J. W. Silverstein and Z. Bai, "On the empirical distribution of eigenvalues of a class of large dimensional random matrices," Journal of Multivariate analysis, vol. 54, no. 2, pp. 175-192, 1995.

[13] R. Horn and C. Johnson, Matrix Analysis. Cambridge University Press, 1990. 